\title{
The Arg451Cys-Neuroligin-3 Mutation Associated with Autism Reveals a Defect in Protein Processing
}

\author{
Davide Comoletti, ${ }^{1}$ Antonella De Jaco, ${ }^{1}$ Lori L. Jennings, ${ }^{1}$ Robyn E. Flynn, ${ }^{1}$ Guido Gaietta, ${ }^{2}$ Igor Tsigelny, ${ }^{1}$ \\ Mark H. Ellisman, ${ }^{2}$ and Palmer Taylor ${ }^{1}$ \\ Departments of ${ }^{1}$ Pharmacology and ${ }^{2}$ Neurosciences and National Center For Microscopy and Imaging Research, University of California, San Diego, La \\ Jolla, California 92093
}

The neuroligins are a family of postsynaptic transmembrane proteins that associate with presynaptic partners, the $\beta$-neurexins. Neurexins and neuroligins play a critical role in initiating formation and differentiation of synaptic junctions. A recent study reported that a mutation of neuroligin-3 (NL3), an X-linked gene, was found in siblings with autistic spectrum disorder in which two affected brothers had a point mutation that substituted a Cys for Arg451. To characterize the mutation at the biochemical level, we analyzed expression and activity of the mutated protein. Mass spectrometry comparison of the disulfide bonding pattern between the native and the mutated proteins indicates the absence of aberrant disulfide bonding, suggesting that the secondary structure of the mutated protein is conserved. However, the mutation separately affects protein expression and activity. The Cys mutation causes defective neuroligin trafficking, leading to retention of the protein in the endoplasmic reticulum. This, in turn, decreases the delivery of NL3 to the cell surface. Also, the small fraction of protein that reaches the cell membrane lacks or has markedly diminished $\beta$-neurexin-1 (NX1 $\beta$ ) binding activity. Other substitutions for Arg451 allow for normal cellular expression but diminished affinity for NX1 $\beta$. Our findings reveal a cellular phenotype and loss of function for a congenital mutation associated with autistic spectrum disorders.

Key words: neuroligin; neurexin; autism; cell adhesion proteins; thiol-retention; trafficking

\section{Introduction}

Autism is a developmental brain disorder characterized by a general inability to form reciprocal social interactions, severe impairment in verbal and nonverbal communication, and a markedly restricted repertoire of activities and interests. The incidence of the autism spectrum disorders is currently estimated at 1 in 1000 children (Folstein and Rosen-Sheidley, 2001). Familial recurrence of the disorder is $\sim 100$-fold higher than in the general population, and concordance rate among monozygotic twins is between 70 and $90 \%$ but close to $0 \%$ in dizygotic twins, thus indicating a strong genetic component in the disease (VeenstraVanderweele et al., 2003). A recent study describes mutations of two X-linked homologous genes encoding neuroligin-3 (NL3) and neuroligin-4 (NL4) in two sets of siblings with autism spectrum disorders. Whereas the NL4 mutation results in a prematurely truncated protein, the mutation of the NL3 gene is a single nucleotide polymorphism that encodes for Cys instead of Arg451 (Jamain et al., 2003).

The neuroligins form a family of five cell adhesion proteins that are widely expressed in brain and in selected locations out-

Received Feb. 9, 2004; revised March 31, 2004; accepted April 13, 2004.

This work was supported by United States Public Health Service Grants P42-ES-10337 and R37 GM-18360 (P.T.) and National Institutes of Health Grant P41 RR04050 (M.H.E.). We thank Dr. T. C. Südhof for the NL1 and NL3 constructs.

Correspondence should be addressed to Palmer Taylor, Department of Pharmacology, University of California, San Diego, 9500 Gilman Drive, La Jolla, CA 92093-0636. E-mail: pwtaylor@ucsd.edu.

DOI:10.1523/JNEUROSCI.0468-04.2004

Copyright $\odot 2004$ Society for Neuroscience $\quad$ 0270-6474/04/244889-05\$15.00/0 side the CNS (Ichtchenko et al., 1996; Philibert et al., 2000; Bolliger et al., 2001). The association between the $\beta$-neurexin-1 $(\mathrm{NX} 1 \beta)$ and the neuroligins appears to be $\mathrm{Ca}^{2+}$ dependent and selectively blocked by insertion of an alternatively spliced region in NX1 $\beta$ (Ichtchenko et al., 1995, 1996; Nguyen and Südhof, 1997). The neurexins are a family of neuronal cell surface proteins composed of three genes containing two independent promoters, resulting in the expression of the longer $\alpha$-neurexins and the shorter $\beta$-neurexins (Missler et al., 1998).

Neurexin and neuroligin trigger presynaptic differentiation in vitro (Scheiffele et al., 2000; Dean et al., 2003), and, in the presence of glutamate receptors, neuroligin-1 (NL1) was shown to form functional excitatory synapses with heterologous cell types (Fu et al., 2003), becoming attractive candidate proteins implicated in the formation and/or maintenance of synaptic recognition patterns in developing brain. Disruption of postnatal synaptic plasticity attributable to neurexin or neuroligin mutations might result in developmental syndromes such as autism spectrum disorders (Zoghbi, 2003). Recombinant soluble NL1 associates with recombinant soluble $\mathrm{NX} 1 \beta$, demonstrating that the NX1 $\beta$ recognition site is completely contained in the $\alpha / \beta$ hydrolase fold domain and that associated proteins are not necessary for the complex formation (Comoletti et al., 2003). Hoffman et al. (2004) showed that the NL1 pattern of disulfide bonding follows that of the $\alpha / \beta$-hydrolase fold family, in which Cys286 in NL1 remains unpaired. Using recombinant rat proteins, we analyzed cellular expression and binding activity of NL3 with Cys substituted for Arg451. Our data suggest that, despite 
the presence of two potential unpaired cysteines, the secondary structure of NL3 remains intact. Nevertheless, the Arg451 to Cys mutation curtails delivery of NL3 to the cell surface, but substitution of Arg451 with Glu and Thr does not influence proper delivery of the protein but virtually abolishes NX1 $\beta$ binding. These data describe a cellular phenotype and loss of function for a congenital mutation associated with autistic spectrum disorders.

\section{Materials and Methods}

Plasmids and mutagenesis. The extracellular domain of the rat NX1 $\beta$ protein between residues 48 and 300 was fused to a C-terminal end of glutathione $S$-transferase (GST) using a pGEX-6P-3 plasmid that permits site-specific cleavage and simultaneous NX1 $\beta$ purification and production in Escherichia coli BL21 cells (Stratagene, San Diego, CA). cDNAs encoding rat NL1 and NL3 were subcloned into FLAG-tagged vector (Sigma, St. Louis, MO) for detection and purification (Comoletti et al., 2003). Briefly, both NL1 and NL3 encode the FLAG octapeptide at their $\mathrm{N}$ termini, a linker peptide of 10 residues, followed by the NL sequence beginning at $\mathrm{Gln} 46$ and Ala35, respectively. Soluble mutants were also constructed by introducing stop codons at Tyr692 in NL1 and Tyr640 in NL3, generating the proteins NL1-691 and NL3-639 respectively.

Cell culture and transfections. HEK293 cells were maintained at $37^{\circ} \mathrm{C}$ and $10 \% \mathrm{CO}_{2}$ in DMEM containing $10 \%$ fetal bovine serum and were periodically tested to ensure the absence of mycoplasma. Cells were transfected with the plasmid and selected by growth in G418 (Geneticin; Sigma) (Comoletti et al., 2003).

$N L 1$ and NX1 $\beta$ expression and purification. To purify the soluble NX1 $\beta$ fusion protein, 200 $\mathrm{ml}$ of bacterial extract containing GST-NX1 $\beta$ fusion protein was affinity purified using glutathione Sepharose 4B (Amersham Biosciences, Piscataway, NJ), and the GST domain was cleaved using PreScission protease (Amersham Biosciences). To purify soluble neuroligins, tissue culture medium containing expressed NL1 and NL3 was passed over an M2 anti-FLAG affinity column (Sigma). The protein was eluted, concentrated to $0.5-1 \mathrm{ml}$, and stored at $4^{\circ} \mathrm{C}$. To purify NLs from cell extracts, $80-10010 \mathrm{~cm}$ dishes of stably transfected cells were grown to confluence. The cells were then harvested in cold PBS, centrifuged, and resuspended in 30 $\mathrm{ml}$ of cold lysis buffer (PBS and $0.1 \%$ Triton $\mathrm{X}-100)$. The solution was centrifuged twice at $15,000 \times \mathrm{g}$ for $15 \mathrm{~min}$ at $4^{\circ} \mathrm{C}$. The clear supernatant containing all of the soluble proteins was incubated at $4^{\circ} \mathrm{C}$ with $\mathrm{M} 2$ anti-FLAG affinity gel, washed, and eluted with FLAG peptide (Comoletti et al., 2003).

Mass spectrometry. NL1-691 wild-type, C286A-NL1-691, C286A/ R473C-NL1-691 (CA/RC-NL1-691), NL3-639, and R471C-NL3-639 were prepared for mass spectrometric analysis using the following conditions. NLs $(50 \mu \mathrm{g})$ at $1 \mathrm{mg} / \mathrm{ml}$ protein in $50 \mathrm{~mm} \mathrm{NH}_{4} \mathrm{HCO}_{3}$, buffer $\mathrm{pH}$ 7.8 , were incubated with $50 \mathrm{~mm}$ iodoacetamide for $2 \mathrm{hr}$ at $37^{\circ} \mathrm{C}$, before the addition of $6 \mathrm{~m}$ guanidine- $\mathrm{HCl}, \mathrm{pH} 6.5$, and incubation for $2 \mathrm{hr}$ at $37^{\circ} \mathrm{C}$. The alkylating reagent and denaturant were removed by spin filtering using Microcon-30 devices (Millipore, Bedford, MA) and exchanged to $50 \mathrm{~mm} \mathrm{NH}_{4} \mathrm{HCO}_{3}$, buffer $\mathrm{pH}$ 7.8. Digestion of NLs with protease and removal of $\mathrm{N}$-linked glycosylation was performed with sequence grade modified trypsin (Promega, Madison, WI) at 1:50 (w/w) of trypsin/NL and $15 \mathrm{mU}$ of PNGase F (Prozyme, San Leandro, CA) for 18 hr at $37^{\circ} \mathrm{C}$

Matrix-assisted laser desorption/ionization time-of-flight mass spectrometry (MALDI-TOF/MS) was performed on a PE Biosystems (Framingham, MA) Voyager DE-STR Biospectrometry workstation.

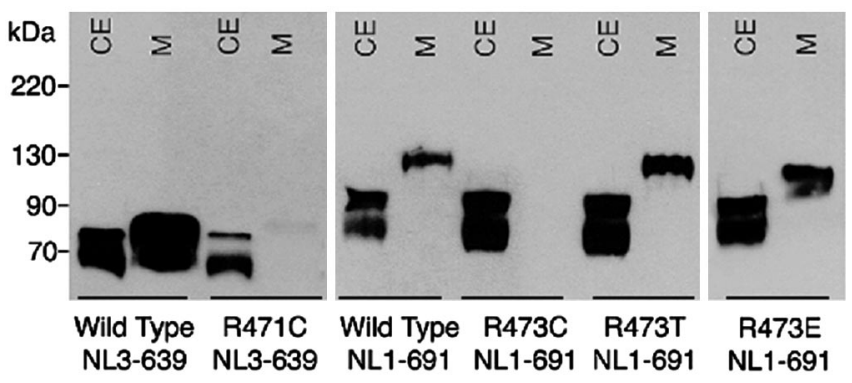

Figure 1. Cellular secretion of wild-type and mutant NL1 and NL3. Immunoblots of the cell extract (CE) and medium (M) from transiently transfected FLAG-tagged NL3 and NL1 mutants truncated at positions 639 and 691, respectively. Sixty hours after transfection, cells and culture media proteins were separated by SDS-PAGE under reducing conditions, and neuroligin proteins were visualized with anti-neuroligin-1/3 monoclonal antibody. Molecular weight standards are shown on the left.
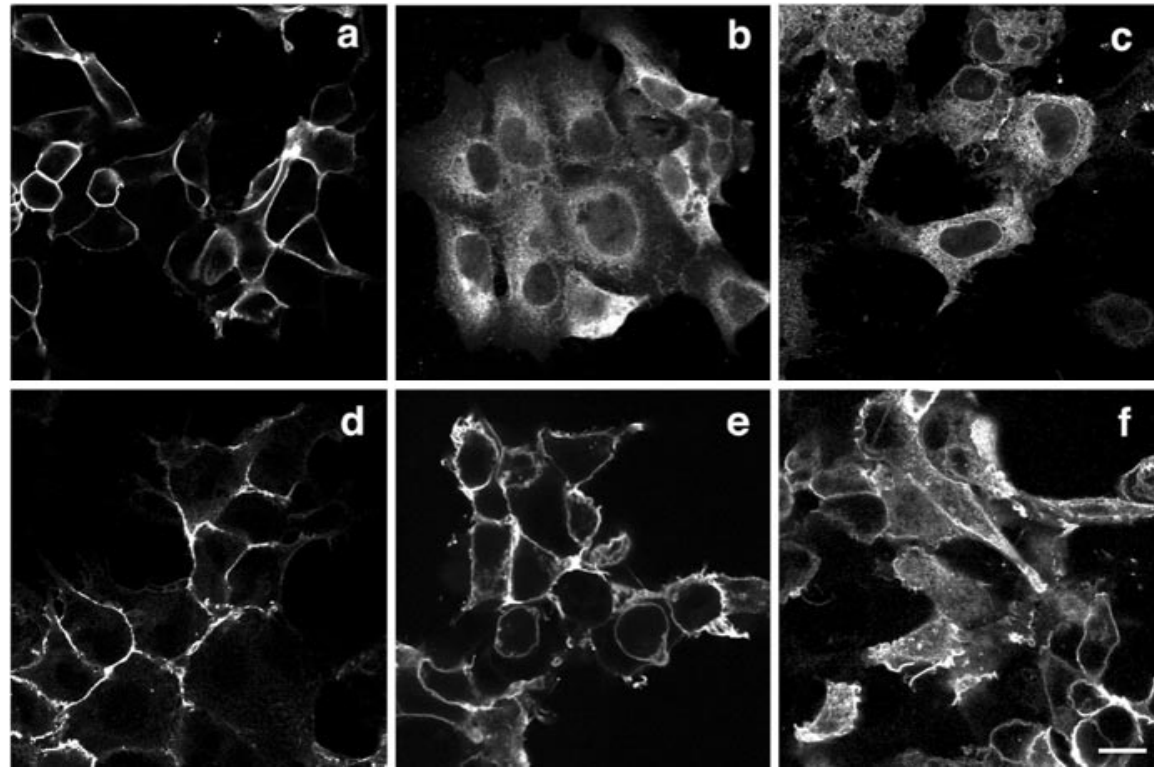

Figure 2. Immunofluorescence detection of the cellular disposition of full-length NL1 and NL3 proteins. Stably transfected cells. Anti-FLAG antibody was used to visualize the neuroligin proteins in cells permeabilized with $0.5 \%$ saponin. $a$, NL1 wild type; b, R473C-NL1; C, CA/RC-NL1; d, R473T-NL1; e, NL3 wild type; $f$, R471C-NL3. Scale bar, $10 \mu \mathrm{m}$.

Trypsinized and de- $N$-glycosylated NLs were mixed with $5 \mathrm{mg} / \mathrm{ml}$ $\alpha$-cyano-4-hydroxycinnamic acid matrix in $50 \%(\mathrm{v} / \mathrm{v})$ acetonitrile and $0.3 \%(\mathrm{v} / \mathrm{v})$ trifluoroacetate, $\mathrm{pH} 2.2$. One microliter aliquots of peptidematrix mixture were spotted on a target plate and dried by slow evaporation. Mass spectra were acquired using delayed extraction conditions in positive-ion reflector and linear modes. External calibration was performed using angiotensin I and ACTH peptides (1-17 and 18-39) for reflector mode and ACTH peptide (7-38) and bovine insulin for calibration in linear mode. Mass spectra were averaged from 256 laser scans, and data were processed with PE Biosystems Grams 3.0 software program.

Surface plasmon resonance analysis of the NL1-NX1 $\beta$ complex. NL1 binding was analyzed at $25^{\circ} \mathrm{C}$ using HEPES buffer, $\mathrm{pH} 7.4$, containing 2 $\mathrm{mM} \mathrm{CaCl}_{2}$ and $0.005 \%(\mathrm{v} / \mathrm{v})$ surfactant P20, on a BIAcore 3000 (BIAcore, Uppsala, Sweden). NX1 $\beta$ was covalently bound to the carboxymethylated dextran matrix of a CM5 chip (Jonsson et al., 1991) at $\sim 3000$ resonance units. Reference data were subtracted from the sample flow channel that did not contain $\beta$-neurexin to obtain specific NX1 $\beta$ binding. NL1 was usually injected over the NX1 $\beta$ surfaces as a set of six concentrations differing by threefold at a flow rate of $50 \mu \mathrm{l} / \mathrm{min}$ to minimize sample diffusion and mass transport limitations. 
Immunofluorescence image acquisition and analysis. Parental HEK293 and NL1 and NL3 stably expressing cells were plated on poly-D-lysinecoated glass coverslips and grown overnight in DMEM. Cells were fixed in $4 \%$ paraformaldehyde-PBS for $20 \mathrm{~min}$, washed, and labeled for immunofluorescence. Briefly, cells were permeabilized with $0.5 \%$ saponin, $1 \% \mathrm{BSA}$, and $10 \mathrm{~mm}$ EDTA in PBS for $5 \mathrm{~min}$ and incubated in blocking buffer (PBS, 2\% normal donkey serum, $0.5 \%$ BSA, and $50 \mathrm{~mm}$ glycine) in the presence of $0.1 \%$ saponin for $30 \mathrm{~min}$. Cells were then incubated for 1 hr in anti-FLAG M2 monoclonal antibody (Sigma) diluted 1:500 in a 1:5 dilution of the blocking buffer. After washing in PBS and then the above buffer, cells were incubated for $1 \mathrm{hr}$ in donkey anti-mouse, Cy5conjugated antibody (Jackson ImmunoResearch, West Grove, PA) diluted 1:100 in buffer. Cells were then washed in PBS containing RNase (Sigma) to remove the excess of secondary antibody and to degrade RNA. Cells were incubated with $5 \mu \mathrm{g} / \mathrm{ml}$ propidium iodide (Sigma) to label nuclei. After washing, cells were mounted in Gelvatol and imaged using an MRC-1024 laser-scanning confocal system (Bio-Rad, Cambridge, MA) coupled to a Zeiss (Oberkochen, Germany) Axiovert $35 \mathrm{M}$ microscope.

Analytical procedures. Western blots were performed using standard procedures. Protein concentrations were estimated by Bradford assay. Antibody anti-neuroligin-1/3 were purchased from Synaptic System (Göttingen, Germany).

\section{Results}

Expression and characterization of truncated neuroligins

Because rat and human NL3 sequences are virtually identical $(99.8 \%)$ and rat NL3 shares $74.1 \%$ amino acid identity with rat NL1 in the extracellular domain, we used NL1 and NL3 rat proteins with mutations at Arg473 and Arg471 respectively, to study the biochemical anomalies caused by the Arg451Cys mutation in humans. The human numbering does not include a 20 aa splice insertion found in rat. Rat NL1 and NL3 cDNAs were truncated to generate the proteins NL1-691 and NL3-639, lacking both the transmembrane and cytoplasmic domains. The 691 truncation occurs just before the transmembrane span, whereas that at 639 also removes an $O$-glycosylation domain that connects $\alpha / \beta$ hydrolase fold region to the membrane-spanning sequence. This region was found to have minimal influence on both expression and NX1 $\beta$ binding (Comoletti et al., 2003). Truncated soluble proteins were necessary for both measuring NX1 $\beta$ binding activity and mass spectrometry analysis.

Transfection of the truncated NL1 and NL3 cDNAs, followed by immunoblotting, reveals the protein in both the cell extract and culture medium, indicating that truncated neuroligins are correctly translated, processed, and transported along the proper trafficking pathway for secretion. The proteins are fully glycosylated, correctly folded, and functional (Comoletti et al., 2003; Hoffman et al., 2004).

\section{Expression and characterization of the mutated soluble neuroligins}

Mutation of the conserved Arg451 to Cys virtually eliminated secretion of soluble NL1 and NL3 into the medium (Fig. 1), indicating that trafficking or folding of the mutated protein might be defective. To ascertain whether the critical alteration was general substitution for Arg or its specific replacement with Cys, two other mutants were made: R473T-NL1-691, to replace Cys with a residue of similar size and polarity but unable to form disulfide bridges, and R473E-NL1-691 to reverse the charge of Arg451. Both mutants were secreted into the culture medium, demonstrating that only the Cys473 substitution disrupted neuroligin targeting to the cell surface (Fig. 1).

\section{Immunofluorescence analysis of full-length neuroligins}

Neuroligins, as synaptic proteins with a single transmembrane span, are targeted to the cell membrane. To ensure that truncation of the protein in combination with the Arg mutation did not influence its trafficking, full-length neuroligins were expressed for an immunofluorescence cell-based assay. On the basis of protein expression profiles, we hypothesized that the newly introduced cysteine could form spurious disulfide bonds with other cysteines, preferentially with the single unpaired Cys286 found naturally in all four members of the NL family (Ichtchenko et al., 1996). To test this possibility, Cys286 was substituted with an Ala in wild type and the R473C-NL1 mutant protein, generating CA/RCNL1. Stably transfected cells permeabilized with saponin and probed with anti-FLAG antibody showed that wild-type NL3 and NL1, as well as R473T-NL1, translocated normally to the cell surface, presenting indistinguishable patterns of expression. Consistent with immunoblotting data, both R473C-NL1 and R471C-NL3 were retained in the cytoplasm. On some cells, R471C-NL3 was visible on the cell membrane, displaying fewer restrictions in retention than in mutated NL1. The residual secretion to the cell surface is possibly attributable to an intrinsically higher level of expression of the recombinant NL3. The double-mutant CA/RC-NL1 did not show sig-
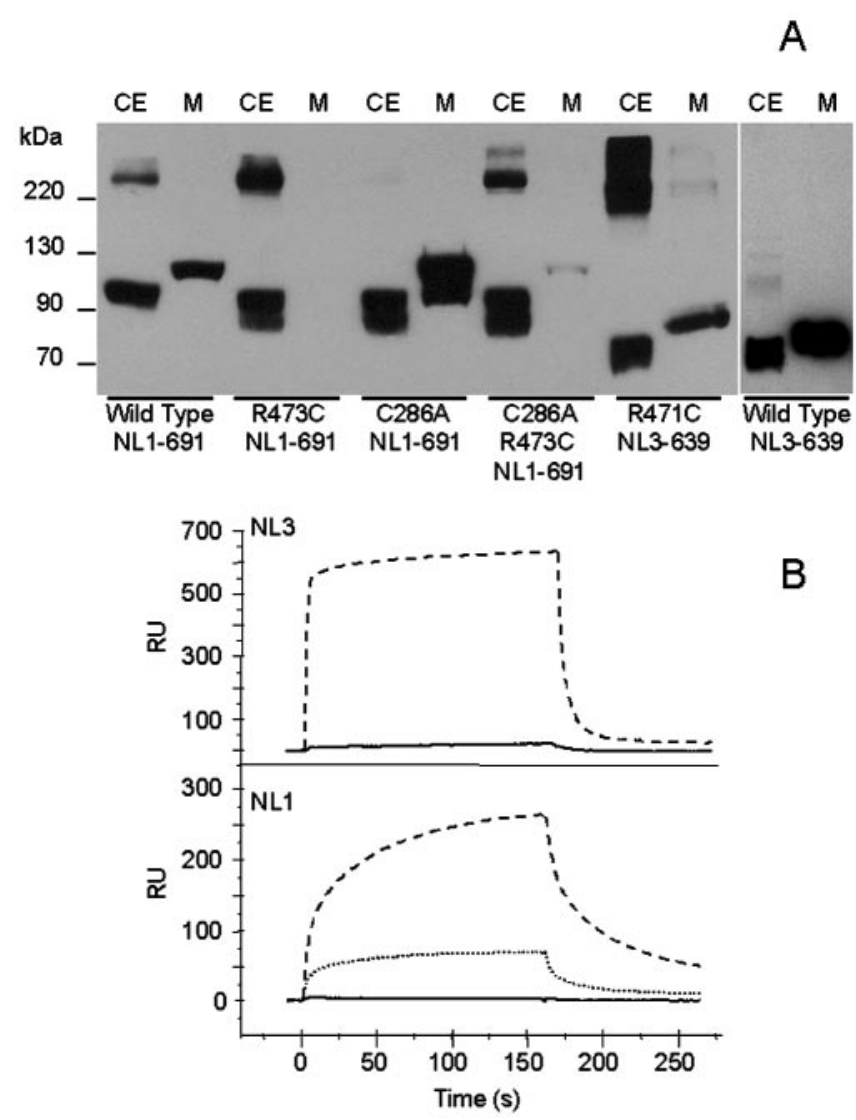

Figure 3. A, Comparison of NL1 and NL3 proteins resolved on SDS-PAGE gel under nonreducing conditions. Immunoblots of the cell extract (CE) and medium (M) of transiently transfected NL1 and NL3 truncated mutants. The samples were prepared in the absence of $\beta$-mercaptoethanol. Other conditions were identical to those in Figure 1. $B$, Surface plasmon resonance binding analysis of soluble neuroligins. Top, NL3-639 and R471C-NL3-639 (3 $\mu \mathrm{m})$ were injected on the same NX1 $\beta$ surface, and binding was measured over a 160 sec interval, followed by a wash period of $160 \mathrm{sec}$. Dashed line, NL3-639; continuous line, R471C-NL3. Bottom, NL1-691, R473T-NL1-691, and R473E-NL1-691 (111 nм) were injected on the same NX1 $\beta$ surface using the same parameters as for NL3. Dashed line, NL1-691 wild type; dotted line, R473T-NL1-691; continuous line, R473E-NL1-691. RU, Resonance units 
nificant improvement in cell surface translocation when compared with both R473CNL1 or R471C-NL3 proteins (Fig. 2), indicating that abnormal disulfide bonding between the substituted Cys at 471 or 473 and Cys286 is not involved in the defective processing.

\section{Electrophoresis under nonreducing conditions}

To ascertain whether the mutated Cys is implicated in intermolecular Cys bonding with either neuroligin itself or heterologous endoplasmic reticulum (ER) resident proteins, the cell lysate and culture medium were subjected to SDS-PAGE in the absence of $\beta$-mercaptoethanol, followed by immunoblotting. In nonreducing conditions, expression of the exported proteins appears to be inversely related to the abundance of intracellular protein aggregates (Fig. 3A). Consistent with the expression pattern found in immunofluorescence, both R473C-NL1-691 and R471C-NL3639 were essentially retained intracellularly. On the contrary, C286A-NL1-691 showed no association with other proteins but optimal extracellular expression. CA/RC-NL1-691 was almost completely retained intracellularly, indicating that the abnormal phenotype of the NL1 was not reversed by eliminating the potential for formation of an additional cysteine loop. Consistent with immunofluorescence data, this result suggests that solvent exposure of the cysteine is critical for retention within the cell.

The components of the aggregate of NL1 are unknown, but their prevalence in nonreducing conditions indicates that intermolecular disulfide bonding is involved (Fig. 3A). Whether the protein was incorrectly folded or subjected to thiol-mediated retention in the endoplasmic reticulum is thus far unresolved.

\section{Mass spectrometric analysis of the soluble NL1 and NL3 proteins}

We reported recently the determination of the disulfide bonding pattern of NL1-691 using mass spectrometry (Hoffman et al., 2004). Respectable expression levels of NL3 enabled us to purify by affinity chromatography both NL3-639 and R471C-NL3-639 from culture medium of stably transfected cells. A small amount of C286A/R473C-NL1-691 was also purified from culture medium, whereas this was not possible with R473C-NL1-691. Tryptic digests of purified de- $N$-glycosylated NL1-691, C286A/ R473C-NL1-691, NL3-639, and R471C-NL3-639 were resolved by MALDI-TOF/MS to establish disulfide pairings. The small fractions of R471C-NL3-639 and C286A/R473C-NL1-691 that are secreted into the medium display the same disulfide bonding pattern as the wild-type proteins (for NL3 data, see Table 1), indicating that the exported proteins have the same secondary structure. In contrast, R473C-NL1-691 protein, purified from cellular lysates, revealed several peptides not attributable to neuroligin in the mass spectral profiles. Copurification with other protein(s) suggests that R473C-NL1-691 retained in the cell is covalently associated with cytoplasmic proteins, perhaps chaperones, as indicated by the large aggregates observed by immunoblotting in nonreducing conditions.

\section{Surface plasmon resonance binding analysis of the mutated proteins}

Analysis of NX1 $\beta$ binding by surface plasmon resonance showed R471C-NL3-639 to have $\sim 50$-fold less NX1 $\beta$ binding than wild type (Fig. 3B, top), whereas for R473T-NL1-691, total binding was approximately threefold less than wild type. Moreover, R473E-NL1-691 strongly decreased NX1 $\beta$ binding, indicating that the homologous Arg in human NL3 also plays a role in recognition of NX1 $\beta$ or overall conformation of neuroligin (Fig. $3 B$, bottom), although the contact surface responsible for the neuroligin-neurexin association remains unknown.

\section{Discussion}

Although substantial evidence has accrued for a genetic linkage to autism spectrum disorders, the absence of an altered function in a defined gene product is a major limitation in understanding the pathogenesis of the disorder. However, the recent discovery of NL3 and NL4 as possible target genes for autism disorders (Jamain et al., 2003), whose malfunction could give rise to aberrant development in the nervous system (Zoghbi, 2003), raises new hopes for early diagnosis of autism and possible therapeutic interventions. Using a recombinant DNA expression system for protein production, mass spectrometric analysis for characterization of the gene product, surface plasmon resonance binding assays, and cell biological analyses of protein biosynthesis, we made several critical observations on how NL3 polymorphisms, discovered in man, could explain their association with autism spectrum disorders.

Introduction of a single Cys in the C-terminal region of the extracellular domain of the NL1 or NL3 uniquely compromises the export of the neuroligins to their cell membrane location because they appear to be retained in the endoplasmic reticulum. In contrast, Thr or Glu substitutions allow the neuroligins to be correctly processed in biosynthesis. However, the pattern of cysteine pairings observed by mass spectrometry suggests that the secreted fraction of the protein is correctly folded. By analysis of migration in reducing versus nonreducing conditions of SDSPAGE and by analysis of mass spectrometry data, the protein retained intracellularly appears disulfide linked with other cytoplasmic proteins, possibly chaperones. Thus far, the precise mechanisms involved in defective trafficking and diminished NX1 $\beta$ binding are not understood. Nevertheless, our data indicate that a global misfolding of the extracellular domain of the neuroligins during biosynthesis and altered secondary structure are not responsible. Rather, the data point toward two distinct mechanisms. First, the mutated Cys may become an ER retention signal for NL3, preventing the protein to reach the cell surface. A thiol retention system has been described in a variety of cell types (Kerem et al., 1993; Reddy et al., 1996), including HEK293 cells, and involves formation of reversible disulfide bonds between ER chaperons and an exposed free Cys residue (Anelli et al. 2003). This mechanism is believed to monitor the assembly status by 
ensuring intermolecular disulfide bonds formation of multimeric proteins such as secretory IgM assembly. On the basis of the available homology model (Hoffman et al., 2004), Cys at position 451 is exposed to solvent and could act as a retention signal similar to Cys575 in the secretory $\mu$ chain of IgM polymers (Davis and Shulman, 1989; Sitia et al., 1990).

Second, the secreted NL3 and NL1 mutated at positions 471 and 473 , respectively, compromise NX1 $\beta$ binding capacities, implicating this region in the neurexin-neuroligin association. Because Arg471 is highly conserved in the neuroligins and acetylcholinesterases among all species (from fruit fly to human) and is located in a predicted EF-hand region (Tsigelny et al., 2000), Jamain et al. (2003) speculated that R451C might modify the binding of neuroligin to NX1 $\beta$, compromising the $\mathrm{Ca}^{2+}$ dependence of the association. Such a mechanism could be at play in diminishing NX1 $\beta$ affinity for the mutant neuroligin. However, the retention in the endoplasmic reticulum appears to be unique to the Cys substitution found in the single base mutation in NL3. The association of the autism spectrum disorders with the neuroligin gene family (Jamain et al., 2003; Laumonnier et al., 2004) and our demonstration of aberrant cellular processing with the Cys substitution and diminished affinity for neurexin with several other neuroligin mutants provide an important lead into the functions of proteins involved in the disorder. Certainly, mutations in the diverse neuroligin family and other proteins forming heterologous cell contacts in the nervous system warrant additional investigation.

\section{References}

Anelli T, Alessio M, Bachi A, Bergamelli L, Bertoli G, Camerini S, Mezghrani A, Ruffato E, Simmen T, Sitia R (2003) Thiol-mediated protein retention in the endoplasmic reticulum: the role of ERp44. EMBO J 22:5015-5022.

Bolliger MF, Frei K, Winterhalter KH, Gloor SM (2001) Identification of a novel neuroligin in humans which binds to PSD-95 and has a widespread expression. Biochem J 356:581-588.

Comoletti D, Flynn RE, Jennings LL, Chubykin A, Matsumura T, Hasegawa H, Südhof TC, Taylor P (2003) Characterization of the interaction of a recombinant soluble neuroligin-1 with neurexin-1beta. J Biol Chem 278:50497-50505.

Davis AC, Shulman MJ (1989) IgM-molecular requirements for its assembly and function. Immunol Today 10:118-122.

Dean C, Scholl FG, Choih J, DeMaria S, Berger J, Isacoff E, Scheiffele P (2003) Neurexin mediates the assembly of presynaptic terminals. Nat Neurosci 6:708-716.

Folstein SE, Rosen-Sheidley B (2001) Genetics of autism: complex aetiology for a heterogeneous disorder. Nat Rev Genet 2:943-955.

Fu Z, Washbourne P, Ortinski P, Vicini S (2003) Functional excitatory syn- apses in HEK293 cells expressing neuroligin and glutamate receptors. J Neurophysiol 90:3950-3957.

Hoffman RC, Jennings LL, Tsigelny I, Comoletti D, Flynn RE, Sudhof TC, Taylor P (2004) Structural characterization of recombinant soluble rat neuroligin 1: mapping of secondary structure and glycosylation by mass spectrometry. Biochemistry 43:1496-1506.

Ichtchenko K, Hata Y, Nguyen T, Ullrich B, Missler M, Moomaw C, Sudhof TC (1995) Neuroligin 1: a splice site-specific ligand for beta-neurexins. Cell 81:435-443.

Ichtchenko K, Nguyen T, Südhof TC (1996) Structures, alternative splicing, and neurexin binding of multiple neuroligins. J Biol Chem 271:2676-2682.

Jamain S, Quach H, Betancur C, Rastam M, Colineaux C, Gillberg IC, Soderstrom H, Giros B, Leboyer M, Gillberg C, Bourgeron T (2003) Mutations of the $\mathrm{X}$-linked genes encoding neuroligins NLGN3 and NLGN4 are associated with autism. Nat Genet 34:27-29.

Jonsson B, Lofas S, Lindqvist G (1991) Immobilization of proteins to a carboxymethyldextran-modified gold surface for biospecific interaction analysis in surface plasmon resonance sensors. Anal Biochem 198:268-277.

Kerem A, Kronman C, Bar-Nun S, Shafferman A, Velan B (1993) Interrelations between assembly and secretion of recombinant human acetylcholinesterase. J Biol Chem 268:180-184.

Laumonnier F, Bonnet-Brilhault F, Gomot M, Blanc R, David A, Moizard MP, Raynaud M, Ronce N, Lemonnier E, Calvas P, Laudier B, Chelly J, Fryns JP, Ropers HH, Hamel BC, Andres C, Barthelemy C, Moraine C, Briault S (2004) X-linked mental retardation and autism are associated with a mutation in the NLGN4 gene, a member of the neuroligin family. Am J Hum Genet 74:552-557.

Missler M, Fernandez-Chacon R, Sudhof TC (1998) The making of neurexins. J Neurochem 71:1339-1347.

Nguyen T, Südhof TC (1997) Binding properties of neuroligin 1 and neurexin 1beta reveal function as heterophilic cell adhesion molecules. J Biol Chem 272:26032-26039.

Philibert RA, Winfield SL, Sandhu HK, Martin BM, Ginns EI (2000) The structure and expression of the human neuroligin-3 gene. Gene 246:303310.

Reddy P, Sparvoli A, Fagioli C, Fassina G, Sitia R (1996) Formation of reversible disulfide bonds with the protein matrix of the endoplasmic reticulum correlates with the retention of unassembled Ig light chains. EMBO J 15:2077-2085.

Scheiffele P, Fan J, Choih J, Fetter R, Serafini T (2000) Neuroligin expressed in nonneuronal cells triggers presynaptic development in contacting axons. Cell 101:657-669.

Sitia R, Neuberger M, Alberini C, Bet P, Fra A, Valetti C, Williams G, Milstein C (1990) Developmental regulation of IgM secretion: the role of the carboxy-terminal cysteine. Cell 60:781-790.

Tsigelny I, Shindyalov IN, Bourne PE, Sudhof TC, Taylor P (2000) Common EF-hand motifs in cholinesterases and neuroligins suggest a role for $\mathrm{Ca}^{2+}$ binding in cell surface associations. Protein Sci 9:180-185.

Veenstra-Vanderweele J, Cook Jr E, Lombroso PJ (2003) Genetics of childhood disorders. XLVI. Autism, Pt 5, Genetics of autism. J Am Acad Child Adolesc Psychiatry 42:116-118.

Zoghbi HY (2003) Postnatal neurodevelopmental disorders: meeting at the synapse? Science 302:826-830. 